\title{
Protruding the tongue improves posterior rhinomanometry in obstructive sleep apnoea syndrome
}

\author{
A. Coste, F. Lofaso, M.P. d'Ortho, B. Louis, E. Dahan, R. Peynegre, A. Harf
}

\begin{abstract}
Protruding the tongue improves posterior rhinomanometry in obstructive sleep apnoea syndrome. A. Coste, F. Lofaso, M.P d'Ortho, B. Louis, E. Dahan, R. Peynegre, A. Harf. (C) ERS Journals Ltd 1999.

ABSTRACT: In posterior rhinomanometry (PRM), oropharyngeal pressure is measured using a tube placed between the tongue and the hard palate. For valid results the patient must position the tongue and soft palate so that both the oropharynx and nasopharynx remain open. A high rate of failure of conventional PRM has been reported in normal individuals. In patients with obstructive sleep apnoea syndrome (OSAS), upper airway abnormalities may further increase the failure rate.

This study proposes a modification of the technique in which protrusion of the tongue enhances pressure transmission between the nasopharynx and the mouth.

In eight normal subjects, resistance was similar when measured by both methods. Of 24 OSAS patients, conventional PRM was unsuccessful in 11. In the remaining 13 patients, a significant correlation between the two methods was found, but resistance was lower by "tongue-out" than by conventional PRM, consistent with a decrease, during tongue protrusion, in retropalatal resistance, which is a component of the "nasal" resistance measured by PRM. In 26 OSAS patients, unilateral nasal resistance values measured by "tongue-out" PRM were similar to those measured by anterior rhinomanometry. When the "tongue-out" method was used routinely in 541 snorers, failure rates were $1.1 \%$ in the 272 non-OSAS patients and $3.7 \%$ in the 269 OSAS patients.
\end{abstract}

These results indicate that posterior rhinomanometry with tongue protrusion is a highly effective tool for measuring nasal resistance in snorers. Eur Respir J 1999; 14: 1278-1282.

\author{
Services de Physiologie-Explorations Fon- \\ ctionnelles et d'Oto-Rhino-Laryngologie, \\ Institut National de la Santé et de la \\ Recherche Médicale INSERM U 492, \\ Hôpital Henri Mondor, 94010 Créteil, \\ France.

\section{Correspondence: A. Harf} \\ Service de Physiologie-Explorations \\ Fonctionnelles \\ Hôpital Henri Mondor \\ 94010 Creteil \\ France \\ Fax: 33149812667
}

Keywords: Nasal resistance obstructive sleep apnoea syndrome upper airways

Received: February 221999 Accepted after revision June 301999
Rhinomanometry provided an objective assessment of nasal patency by measuring nasal resistance to airflow, requiring simultaneous determination of transnasal pressure and airflow. In posterior rhinomanometry (PRM), oropharyngeal pressure is measured using a tube inserted through the closed lips and placed between the tongue and the hard palate with airflow measured using a pneumotachograph incorporated in the breathing port of a face mask. The presence of the tube in the mouth can cause discomfort. The patient must be told how to position the tongue so that both the oropharynx and nasopharynx remain open [1]. Failure rates for PRM of up to $30 \%$ have been reported in normal individuals [1-5].

In obstructive sleep apnoea syndrome (OSAS), negative intraluminal airway pressure plays a key role in the destabilization that leads to pharyngeal airway obstruction. Because an increase in nasal resistance is associated with decreased intraluminal airway pressure, elevated nasal resistance has been suggested as a factor in sleep apnoea, although conflicting results have been reported [6-11]. To assist in identifying those OSAS patients most likely to benefit from nasal surgery, PRM was included in the evaluation of sleep apnoea syndrome. Adequate oropharyngeal pressure measurements could not be obtained which was ascribed to the upper airway abnormalities associated with OSAS. These abnormalities may make it difficult to obtain free communication between the mouth and the nasopharynx, a requirement for satisfactory PRM.

Alternatively unilateral nasal resistance can be evaluated by anterior rhinomanometry (ARM), where the pressure in the nasopharynx is recorded via a small tube sealed in an occluded nasal cavity while the subject breathes through the other nostril. Although ARM is easy to perform, it requires that total nasal resistance be calculated using Ohm's law for parallel resistors, which is reliable only for the decongested nose [5].

In this study, a modification of conventional PRM is presented, in which protrusion of the tongue is used to facilitate pressure transmission between the nasopharynx and the mouth. This method was found to be highly effective in OSAS patients.

\section{Materials and methods}

\section{Rhinomanometry}

Conventional methods. Measurements were made according to international recommendations [12]. For PRM, flow measurements were carried out using a transparent plastic face mask fitted with a Fleisch No.1 pneumotachograph (Lausanne, Switzerland). The pneumotachograph was connected to a pressure transducer (Validyne 
MP 45, Northridge, CA, USA; $\pm 2 \mathrm{cmH}_{2} \mathrm{O}$ ). Oropharyngeal pressure was recorded via a tube inserted through the closed lips and placed between the tongue and the hard palate while the patient breathed through the nose. The plastic tube $(0.5 \mathrm{~cm})$ was placed as far back in the mouth as the subject could tolerate. One port of a differential pressure transducer (Validyne MP 45, \pm 70 $\mathrm{cmH}_{2} \mathrm{O}$ ) was connected to the oral tube and the other port to the mask to allow transnasal pressure measurement. PRM is the only method for determining total nasal resistance. When one nostril is sealed off with adhesive tape, it also allows the measurement of unilateral resistance. For ARM, unilateral transnasal pressure was obtained by connecting the pressure transducer to an occluded nasal cavity, while the subject breathed through the other side.

Pressure and flow signals were recorded simultaneously at a rate of $32 \mathrm{~Hz}$ on the hard disk of a microcomputer using the Acknowledge software and device (Biopac Systems Inc., Santa Barbara, CA, USA). With this software, the pressure-time and flow-time curves, together with the pressure-flow curve, can be displayed in real time, allowing visual feedback to the patient from the trace on a monitor. Data were processed immediately after each $30 \mathrm{~s}$ recording to compute resistance as the pressure-flow ratio at a transnasal pressure of $1 \mathrm{cmH}_{2} \mathrm{O}$ [5].

The "tongue out" posterior rhinomanometry method. As shown in figure 1 the patient was instructed to stick out the tongue between the lower edge of a modified mouthpiece and the lower lip. No leakage around the tongue was observed. The position of the tongue could be easily modified as needed, using the visual feedback from the pressure and flow signals displayed on a screen.

A catheter connected to the pressure transducer was inserted through a hole drilled in a stopcock obstructing the cylindrical part of the mouthpiece. The distal end of the catheter remained inside the mouthpiece, so that it could not be obstructed by saliva or by the tongue.

Because of the presence of a mouthpiece, a nasal mask was used instead of a facial mask to record nasal flow. Respironics vinyl masks (Nantes, France) in three different sizes were used. These masks have a soft plastic rim that seals tightly without deforming the nose.

\section{Subjects and protocol}

Comparison of the two posterior rhinomanometry methods in normal subjects. Eight healthy volunteers with no history of nasal disease or trauma and no evidence of septal deviation were recruited from the staff of the authors' department. Their age range was 18-50 yrs. All subjects were informed about the study objectives and were allowed enough time to become familiar with the rhinomanometry procedure. Total nasal resistance was computed first, followed by right and left nasal resistances with the other nostril sealed using adhesive tape. Unilateral resistances were measured to evaluate how well the method assessed high levels of resistance.

In each subject, nasal resistance was recorded three times for each method (conventional and "tongue out") and each condition (total, right, and left resistances), and the averages were used for subsequent analysis. a)

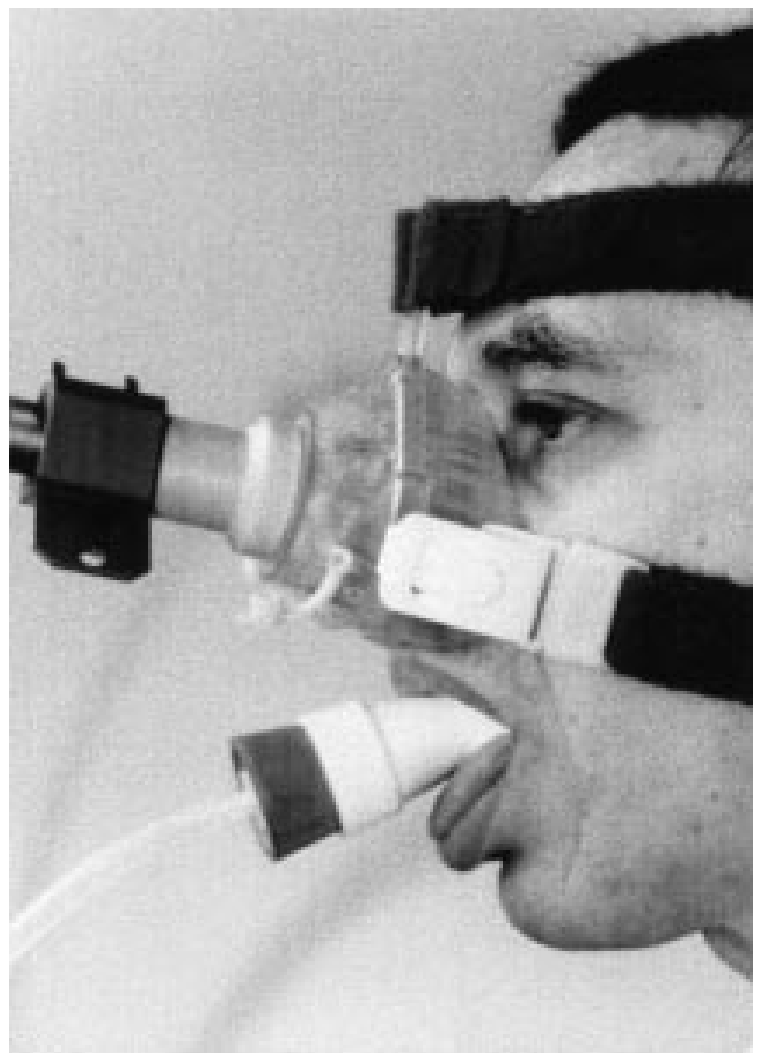

b)

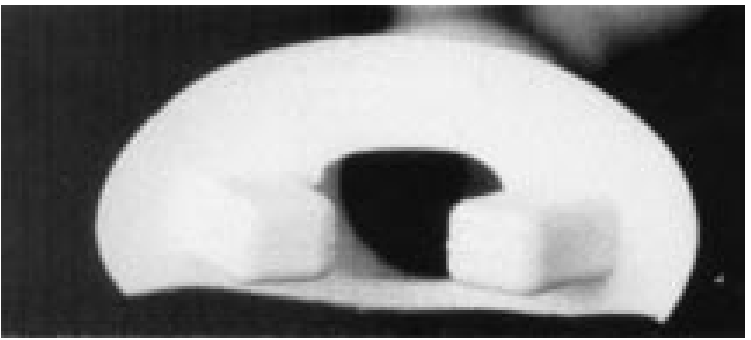

Fig. 1. - During the "tongue out" posterior rhinomanometry method, the patient is instructed to protrude the tongue between the lower edge of the mouth piece and the lower lip (a). A conventional mouthpiece was modified by cutting off part of the flange intended to be placed between the teeth and lower lip (b). The pressure catheter was inserted through a hole drilled in a stopcock obstructing the cylindrical part of the mouthpiece, with the distal end of the catheter remaining inside the mouthpiece. A nasal mask was used to record nasal flow.

Comparison of rhinomanometry methods in obstructive sleep apnoeics. Twenty-four consecutive OSAS patients (mean apnoea-hypopnoea index (AHI) 37 events $\cdot h^{-1}$ ) volunteered to participate in the study. Nasal resistance was measured following the same protocol as for the normal subjects, except that only total nasal resistance was evaluated. The two methods (conventional and "tongue out") were used in random order. Since a high rate of failure occurred with the conventional method (see Results section), the number of trials was limited to six, varying the position of the tongue (inside the mouth) and catheter between trials. Total test duration was $\sim 10 \mathrm{~min}$.

In another group of 26 OSAS patients (mean AHI 32 events $\cdot \mathrm{h}^{-1}$ ), unilateral nasal resistances were measured by the "tongue out" PRM method and by ARM, in random order. 
Failure rate during routine use of the "tongue out" meth$o d$. After the preliminary validation, the "tongue out" method was found to be much easier to perform and it was therefore decided to use it on a routine basis. During a 2-yr period, 541 patients who attended the sleep clinic for snoring and varying degrees of daytime somnolence were investigated. The rate of failure to obtain reliable data in this population was recorded.

\section{Statistics}

Regression analysis was performed to compare the results obtained with the two rhinomanometry methods. In addition, as recommended by BLAND and ALTMAN [13] for the comparison of a new method to an established one, the agreement between the two methods was determined by plotting the difference between the methods against their mean.

\section{Results}

Comparison of the two posterior rhinomanometry methods in normal subjects

In each of the normal subjects, the two rhinomanometry methods provided adequate pressure-flow data allowing computation of total, right and left nasal resistances. Total nasal resistance ranged $1.3-3.0 \mathrm{cmH}_{2} \mathrm{O} \cdot \mathrm{L}^{-1} \cdot \mathrm{s}$, whereas unilateral resistances were usually higher.

Regression analysis showed a highly significant correlation between the two methods ( $\mathrm{y}$ ("tongue out") $=0.96$, $\mathrm{x}$ (conventional) $+0.07, \mathrm{r}=0.99)$. Bland and Altman analysis showed the absence of systematic bias in the measurement (mean \pm SD $2 \pm 8 \%$ ) and showed that the error did not increase with the level of resistance. In two subjects, unilateral nasal resistances were $>10 \mathrm{cmH}_{2} \mathrm{O} \cdot \mathrm{L}^{-1} \cdot \mathrm{s}$ : even at these high levels, the two rhinomanometry methods yielded similar results.

Comparison of the two posterior rhinomanometry methods in obstructive sleep apnoeics

In 11 of the 24 patients, adequate pressure-flow data using the conventional method could not be obtained. In each of these 11 patients, the "tongue out" method gave the expected pattern of pressure and flow signals. A representative set of data is presented in figure 2 .

For the 13 remaining patients, satisfactory pressureflow data were obtained using both methods. As shown in figure 3 , a significant correlation was found between the results of the two methods. In the higher resistance range, however, the conventional method yielded higher values than the "tongue out" method. The mean \pm SD difference between the methods was $13 \pm 15 \%$.

\section{"Tongue out" posterior rhinomanometry versus anterior rhinomanometry in obstructive apnoeics}

It was found that unilateral nasal resistance values provided by the "tongue out" PRM method were similar to those obtained using ARM (data not shown).
Regression analysis showed a highly significant correlation between the two methods (y (PRM) r=0.98); $\mathrm{x}$ $(\mathrm{ARM})+0.2, \mathrm{r}=0.99)$. Bland and Altman analysis showed the absence of systematic bias in the measurement (mean \pm SD $2 \pm 3 \%$ ) and that the error did not increase with the level of resistance. In three subjects, unilateral nasal resistances were $>10 \mathrm{cmH}_{2} \mathrm{O} \cdot \mathrm{L}^{-1} \cdot \mathrm{s}$; even at these high levels, the two rhinomanometry methods yielded similar results.

\section{Rate of failure during routine use of the "tongue out" method}

During a 2-yr period, rhinomanometry was performed in 541 patients with suspected OSAS, using the "tongue out" rhinomanometry method only.

The "tongue out" method gave reliable data in all but three $(1.1 \%)$ of 272 non-OSAS patients with an AHI $<15$ events $\cdot \mathrm{h}^{-1}$. Of 269 OSAS patients with an AHI $\geq 15,10$ (3.7\%) failed the investigation. Mean AHI was similar in these 10 patients and in the other 259 OSAS patients (42 \pm 15 versus $37 \pm 19$, respectively).

\section{Discussion}

The key to successful PRM is to obtain a reliable measurement of transnasal pressure by means of the oral tube. Patients must be trained to keep the soft palate elevated and the upper surface of the tongue away from the palate so as to ensure free communication between the oropharynx and the oral cavity. Insufficient palatal control, together with an unwillingness to suck the tube, seems to be responsible for poor tolerance of the tube. As stated by CoLE [5], "patience and persuasion are required to coach each patient in the suitable positioning of the tongue and the soft palate to maintain patency of both the oropharyngeal and nasopharyngeal orifices, while breathing through the nose". This author reported a failure rate of $15 \%$ in more than 5,000 patients. Similarly, a review by SCHUMAKER [14] indicates that $\sim 20 \%$ of normal individuals have insufficient palatal control to allow pressureflow recordings consistently free of artefacts. In other studies, failure rates have ranged from $8 \%$ (in well-trained individuals) [15] to $50 \%$ (in children) [16]. In a recent study by CANBAY et al. [2], nine of $56(16 \%)$ subjects did not complete the PRM recordings satisfactorily and were therefore excluded from the study. Interestingly, the same group who reported a failure rate of $15 \%$ with conventional PRM suggested that a plethysmograph be used for recording flow in order to leave free access to the face and therefore to obtain better control of the position of the oral tube; with this technique, the failure rate was as low as $1.4 \%$ in 1,000 paediatric patients [17].

To the authors' knowledge, the PRM failure rate in OSAS patients has not been evaluated. In the experience of the authors, conventional PRM is especially difficult to perform in OSAS patients, even by highly-trained technicians. In the group of 24 patients used in the present study to compare conventional versus "tongue out" PRM, reliable data could not be obtained in 11 patients. These failures may have been ascribable to various craniofacial abnormalities and/or upper airway soft tissue abnormalities known to occur in adult OSAS patients [18]. In addition, a large, posteriorly-positioned tongue base contributes to 

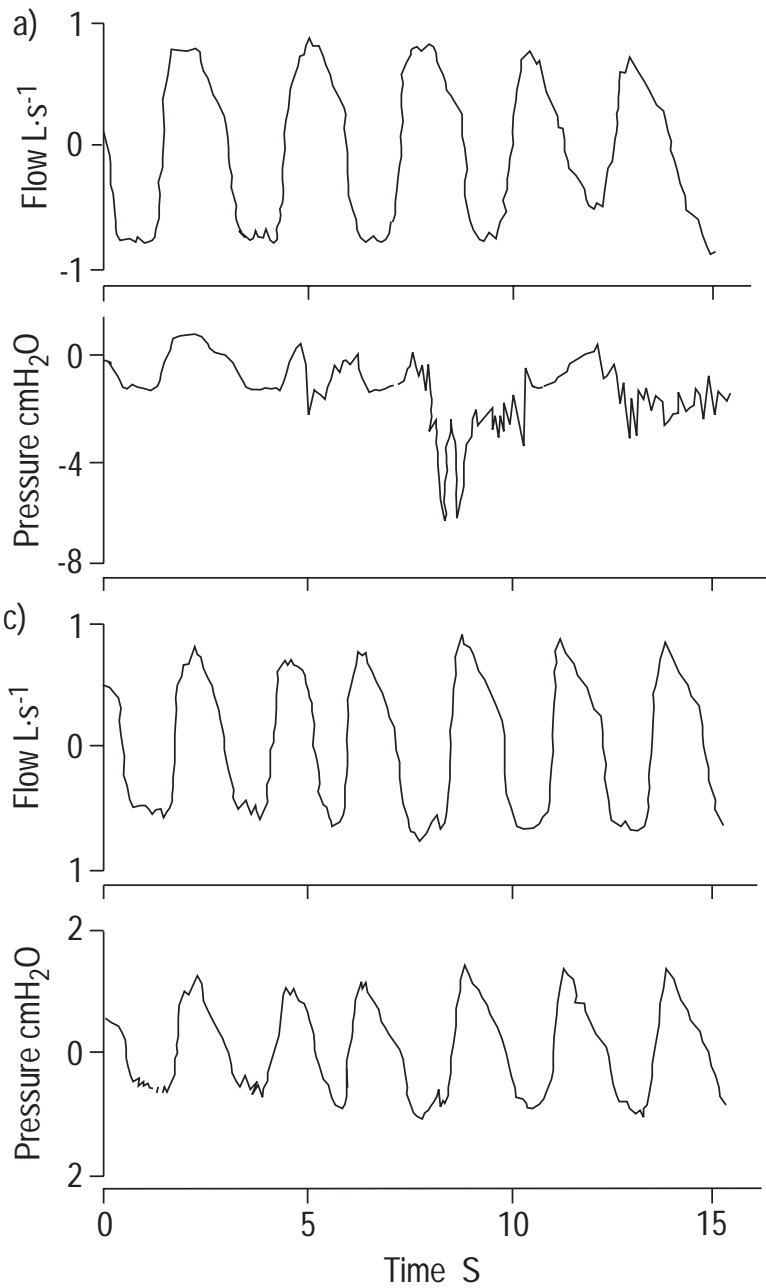

b)
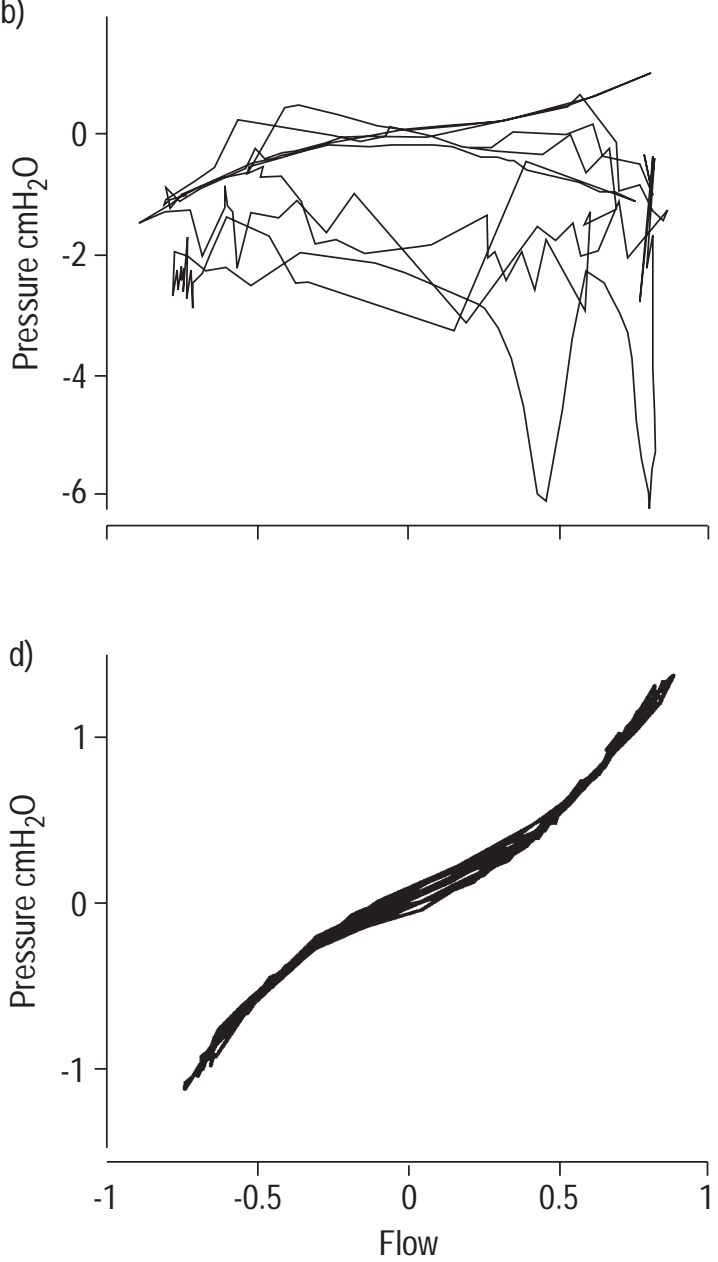

Fig. 2. - Representative data obtained in a patient with obstructive sleep apnoea syndrome during conventional (a, b) and "tongue out" (c, d) posterior rhinomanometry. a, c) Time curves of nasal flow and oral pressure signals; b, d) the same signals presented as XY plots. In this patient, adequate pressureflow relationships were obtained with the conventional methods during a single respiratory cycle, whereas expected patterns of pressure and flow signal were recorded throughout the investigation with the "tongue out" method.

narrowing of the posterior oropharynx. These anatomical factors make it difficult to obtain free communication between the mouth and the nasopharynx in a number of patients with OSAS, leading to failure of pharyngeal pressure measurements during conventional PRM. It was hypothesized that protruding the tongue may improve communication between the mouth and pharynx, thus circumventing the problems raised by the anatomical abnormalities that place the base of the tongue closer to the posterior wall of the pharynx and to the soft palate. A recent study [19] found that protruding the tongue increased the cross-sectional area in the hypopharynx, oropharynx and velopharynx. Furthermore, obese patients had a larger relative increase in the upper airway crosssectional area than did subjects of normal weight.

A sealed mouthpiece was used during rhinomanometry, as previously described [1], which allowed positioning of the oral tube so that it was not in contact with the tongue. It is usually recommended that the tube be held as far back in the mouth as the subject can tolerate [20]. On the other hand, it has also been suggested that a short oral tube is adequate for pharyngeal pressure monitoring provided the position of the tongue allows free commu- nication between the anterior part of the oral cavity and the oropharynx [14]. Since tongue protrusion used in the present study appeared to improve this communication substantially, it was reasoned that pressure measurement could be performed at any site in the oral cavity. It was chosen to perform the pressure measurements within the mouthpiece to avoid contact between the pressure tube and the tongue, thus making it easier for the subject to control the position of the tongue. Furthermore, this method avoids obstruction of the tube by saliva forced into the tube lumen after swallowing.

With the modified PRM method, erratic pressure signals were avoided and reliable measurements obtained in most OSAS patients. In the large series of patients investigated using "tongue out" PRM, the failure rate was only 3.7\%, clearly attesting to the usefulness of this method in such patients.

Resistance was higher by conventional PRM than by "tongue out" PRM in some OSAS patients. PRM measures pressure in the oropharynx and, consequently, the calculated resistance includes any resistive elements located between the atmosphere and the oropharynx during nasal breathing. In normal subjects, the calculated resistance 

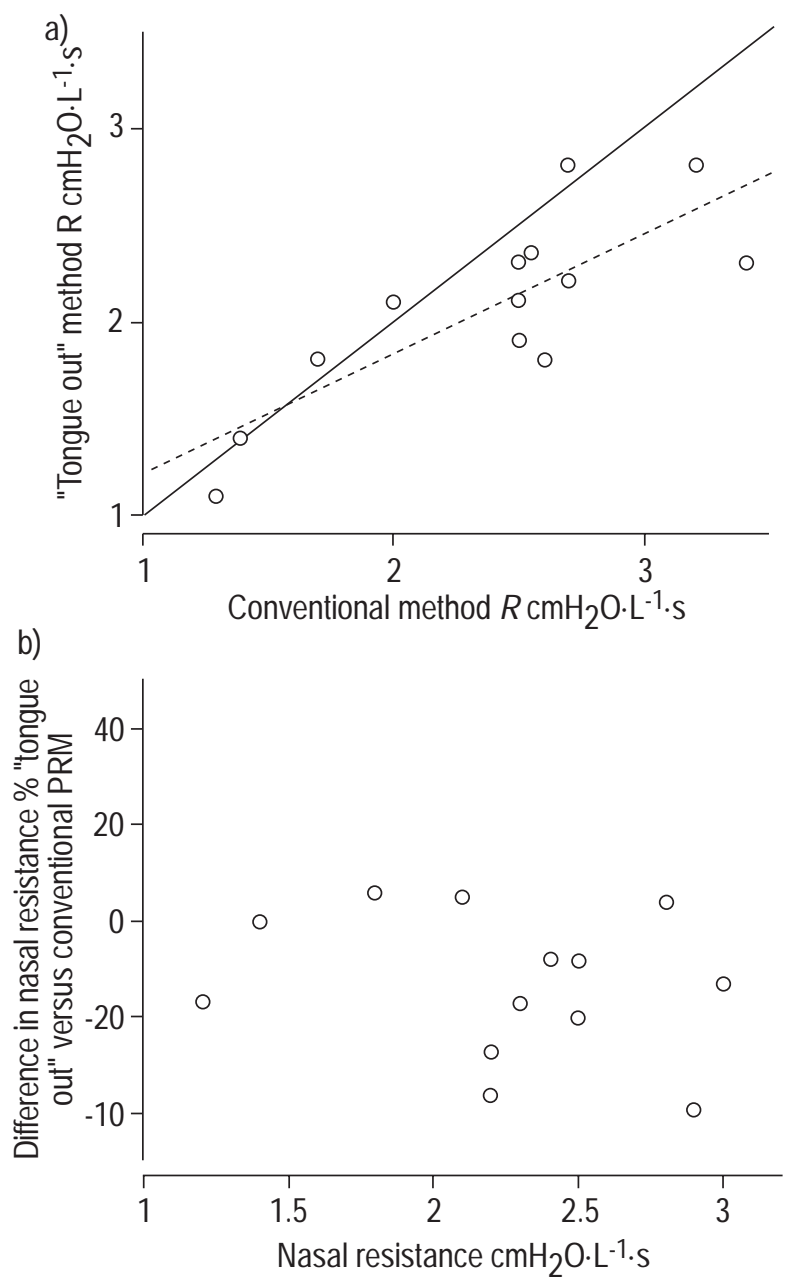

Fig. 3. - Comparison of total nasal resistance measured using the conventional and "tongue out" posterior rhinomanometry (PRM) methods in 13 obstructive sleep apnoea syndrome patients. a) The regression analysis shows a significant correlation between the two methods (y ("tongue out" PRM) $=0.63 \times$ (conventional PRM) $+0.85, \mathrm{r}=0.81)$. The solid and dashed lines are the identity line and the regression line, respectively. Bland and Altman analysis: the mean \pm SD difference between the methods is $13 \pm 15 \%$. In the higher resistance range, the conventional method provided higher values than "tongue out" method.

may be due only to the nose, whereas in OSAS patients, additional resistance may be produced by the anatomical abnormalities of the pharyngeal region. Sticking out the tongue may minimize this additional resistance, thus lowering the calculated resistance as compared to the value obtained during conventional PRM. The present findings that "tongue out" PRM gives unilateral nasal resistance values similar to those obtained with anterior rhinomanometry further supports the fact that the "tongue out" PRM may be especially appropriate for evaluating nasal resistance in patients with increased pharyngeal resistance.

In summary, these data demonstrate that "tongue out" posterior rhinomanometry increases the success rate of nasal resistance measurement compared to conventional posterior rhinomanometry, even in patients with anatomical abnormalities of the pharyngeal region such as the macroglossia or long soft palate commonly present in obstructive sleep apnoea syndrome patients. Although rhinomanometry has been in use for many years, it has not been widely adopted as a routine method in clinical trials, primarily because of the high failure rate of posterior rhinomanometry. The "tongue out" variant investigated in this study may be a substantial improvement in rhinomanometry technique and may provide valuable data regarding the potential role of nasal obstruction in the occurrence of sleep apnoea.

\section{References}

1. Lund VJ. Objective assessment of nasal obstruction. Otolaryngol Clin N Am 1989; 22: 279-290.

2. Canbay EI, Bhatia SN, Orth D. An evaluation of active anterior and posterior rhinomanometry in British adults. Am J Rhinol 1996; 10: 33-37.

3. Gleeson MJ, Youlten LF, Shedon D, et al. Assessment of nasal patency: a comparison of four methods. Clin Otolaryngol 1986; 11: 99-107.

4. Kern EB. Standardization of rhinomanometry. Rhinology 1977; 15: 115-117.

5. Cole P. Rhinomanometry 1988: practice and trends. Laryngoscope 1989; 99: 311-315.

6. Atkins M, Taskar V, Clayton N, Stone P, Woodcock A. Nasal resistance in obstructive sleep apnoea. Chest 1994; 105: 1133-1135.

7. Dayal VS, Phillipson EA. Nasal surgery in the management of sleep apnoea. Ann Otol Rhinol Laryngol 1985; 94: 550-554.

8. Lavie P, Gertner R, Zomer J, Podoshin L. Breathing disorders in sleep associated with "micro arousals" in patients with allergic rhinitis. Acta Otolaryngol (Stockh) 1981; 92: 529-533.

9. Miljeteig H, Hoffstein P, Cole P. The effects of unilateral and bilateral nasal obstruction on snoring and sleep apnoea. Laryngoscope 1992; 102: 1150-1152.

10. Sériès F, St Pierre S, Carrier G. Effects of surgical correction of nasal obstruction in the treatment of obstructive sleep apnoea. Am Rev Respir Dis 1992; 146: 1261-1265.

11. Sériès F, St Pierre S, Carrier G. Surgical correction of nasal obstruction in the treatment of sleep apnoea: importance of cephalometry in predicting outcome. Thorax 1993; 48: 360-363.

12. Clement PAR. Committee report on standardization of rhinomanometry. Rhinology 1984; 22: 151-155.

13. Bland MJ, Altman DG. Statistical methods for assessing agreement between two methods of clinical measurement. Lancet 1986; 8: 307-310.

14. Schumaker MJ. Rhinomanometry. J Allergy Clin Immunol 1989; 83: 711-718.

15. Nolte D, Luder-Luhr I. Comparing measurements of nasal resistance by body plethysmography and by rhinomanometry. Respiration 1973; 30: 31-38.

16. Georgitis JW. The applicability of rhinomanometry in nonatopic children: comparison of three techniques. $J$ Allergy Clin Immunol 1985; 75: 614-620.

17. Cole P, Havas TE. Nasal resistance to respiratory airflow: a plethysmographic alternative to the face mask. Rhinology 1987; 25: 159-166.

18. Ferguson KA, Ono T, Lowe AA, Ryan CF, Fleetham JA. The relationship between obesity and craniofacial structure in obstructive sleep apnoea. Chest 1995; 108: 375-381.

19. Ferguson KA, Love LL, Ryan CF. Effect of mandibular and tongue protrusion on upper airway size during wakefulness. Am J Respir Crit Care Med 1997; 155: 17481754.

20. James DJ, Stidley CA, Mermier CM, Lambert WE, Chick TW, Samet JM. Sources of variability in posterior rhinomanometry. Ann Otol Rhinol Laryngol 1993; 102: 631-638. 